\title{
Formation of perpendicularly aligned sub-10 nm nanocylinders in poly $(N$-dodecyl acrylamide- $b$ - ethylene glycol) block copolymer films by hierarchical phase separation
}

Kohei Matsunaga, ${ }^{\dagger}$ Wataru Kukai, ${ }^{\dagger}$ Manabu Ishizaki, ${ }^{\perp}$ Masato Kurihara,${ }^{\perp}$ Shunsuke Yamamoto, ${ }^{\dagger}$ Masaya Mitsuishi, ${ }^{\neq}$Hiroshi Yabu, \# Shusaku Nagano, ${ }^{\S}$ and Jun Matsui ${ }^{\perp^{*}}$

$\dagger^{\dagger}$ Graduate School of Science and Engineering, ${ }^{\perp}$ Faculty of Science, Yamagata University, 1-4-12

Kojirakawa-machi, Yamagata 990-8560, Japan

\#Institute of Multidisciplinary Research for Advanced Materials, ${ }^{\#}$ WPI-Advanced Institute for

Materials Research, Tohoku University, 2-1-1 Katahira, Aoba-ku, Sendai 980-8577, Japan

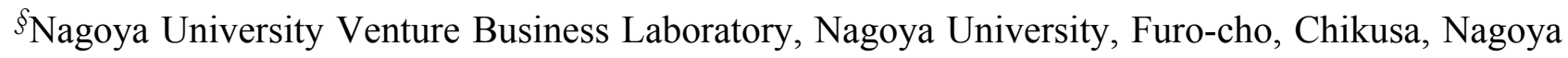
464-8603, Japan

*Author to whom correspondence should be addressed. E-mail: jun_m@sci.kj.yamagata-u.ac.jp 


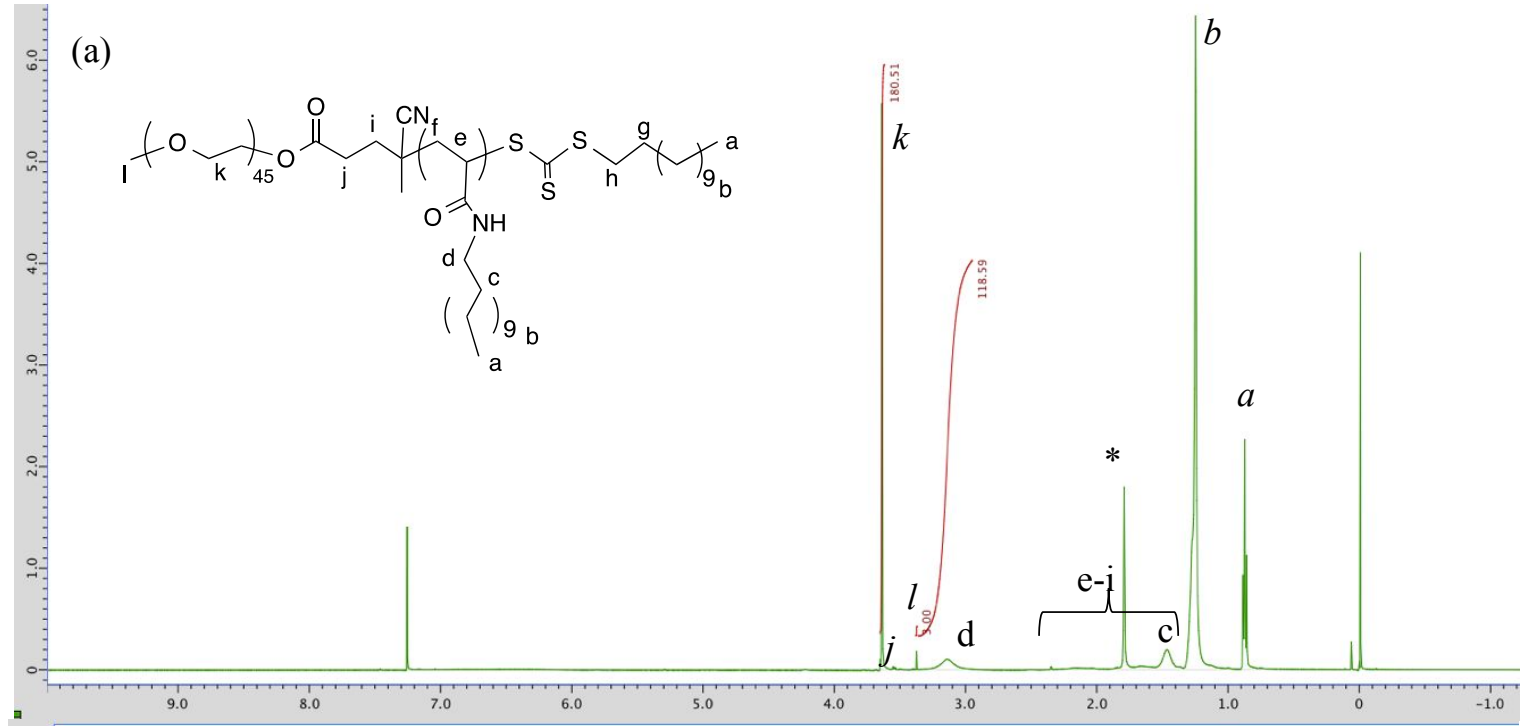

(b)
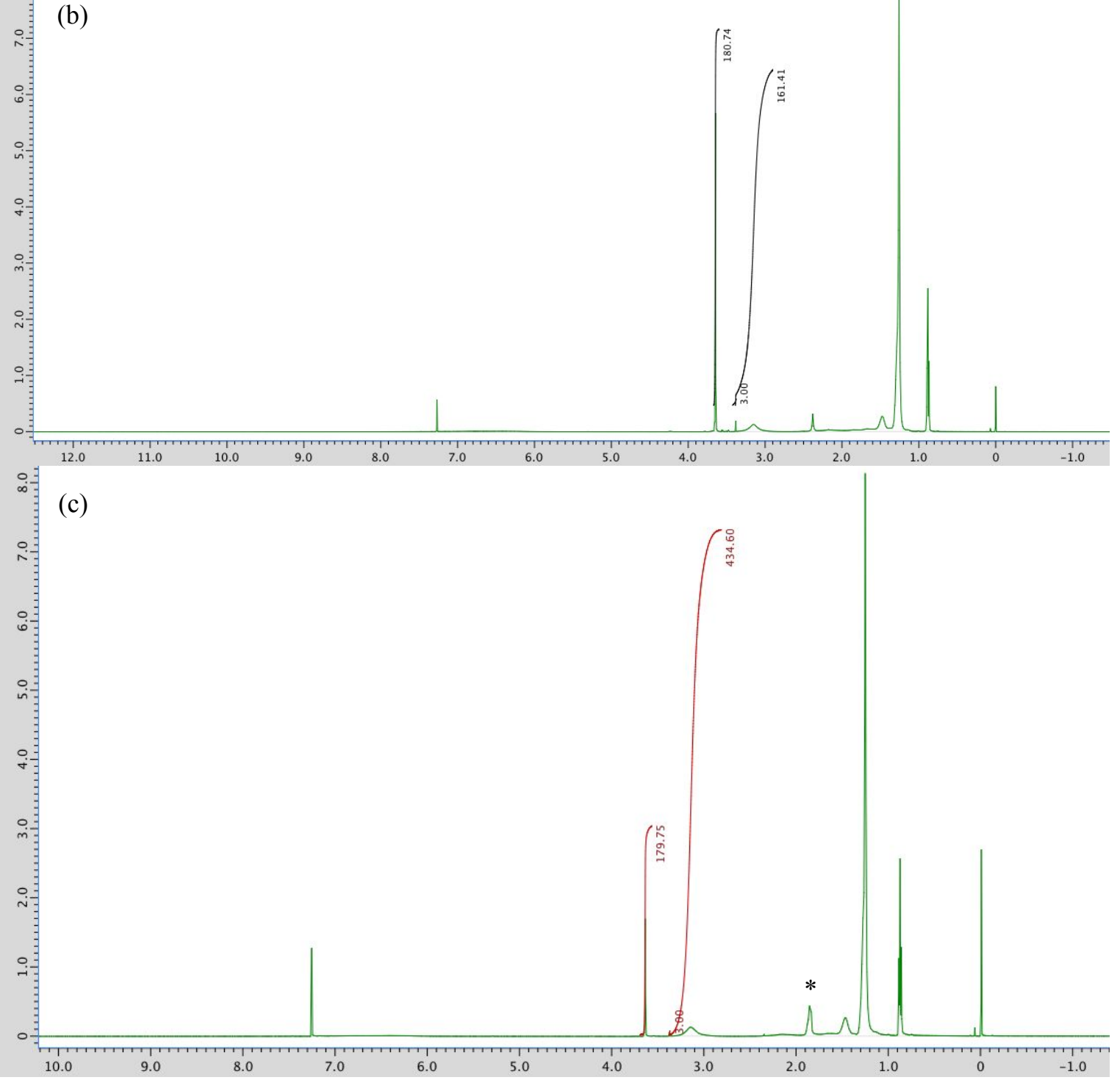

Figure S1 ${ }^{1} \mathrm{H}$ NMR spectra for (a) $\mathrm{pDDA}_{60}-b-\mathrm{PEG}_{45}$, (b) $\mathrm{pDDA}_{80}-b-\mathrm{PEG}_{45}$, and (c) $\mathrm{pDDA}_{218}-b-\mathrm{PEG}_{45}$ * peak was assigned to water hydrogen bonded with PEG groups.

2 


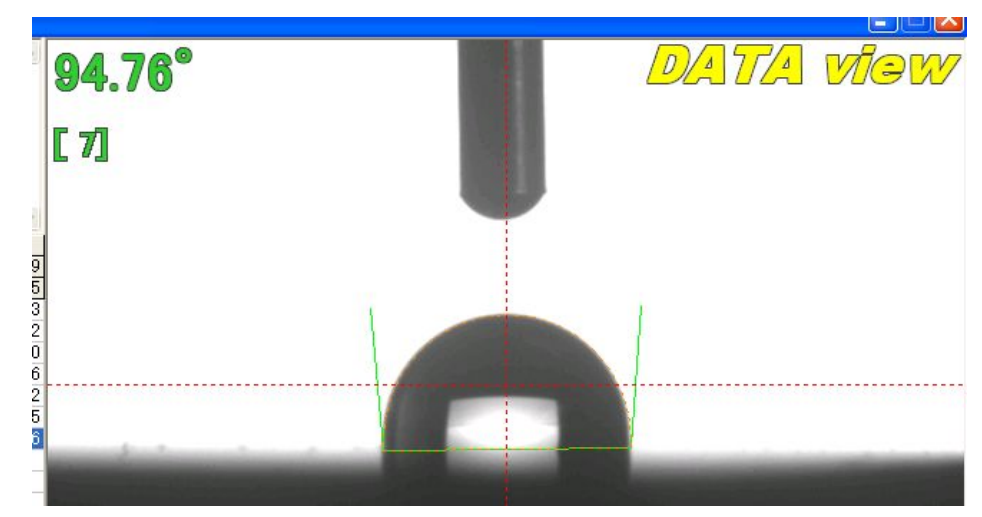

Figure S2 Water contact angle of the surface of thermally annealed $\mathrm{pDDA}_{80}-b-\mathrm{PEG}_{45}$ film

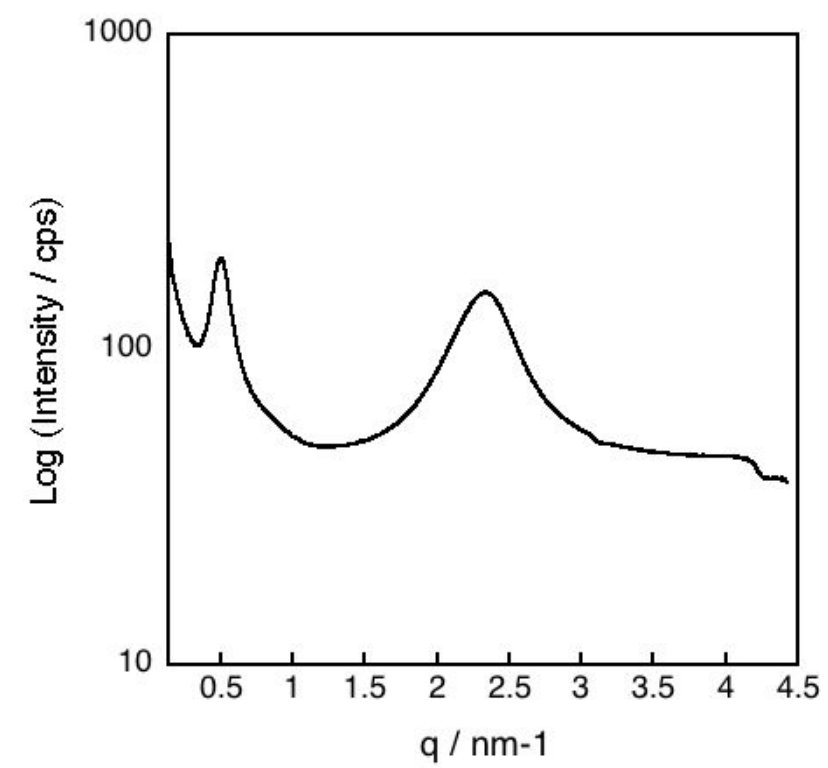

Figure $\mathbf{S 3}$ XRD profile for $\mathrm{pDDA}_{80}-b-\mathrm{PEG}_{45}$ powder 


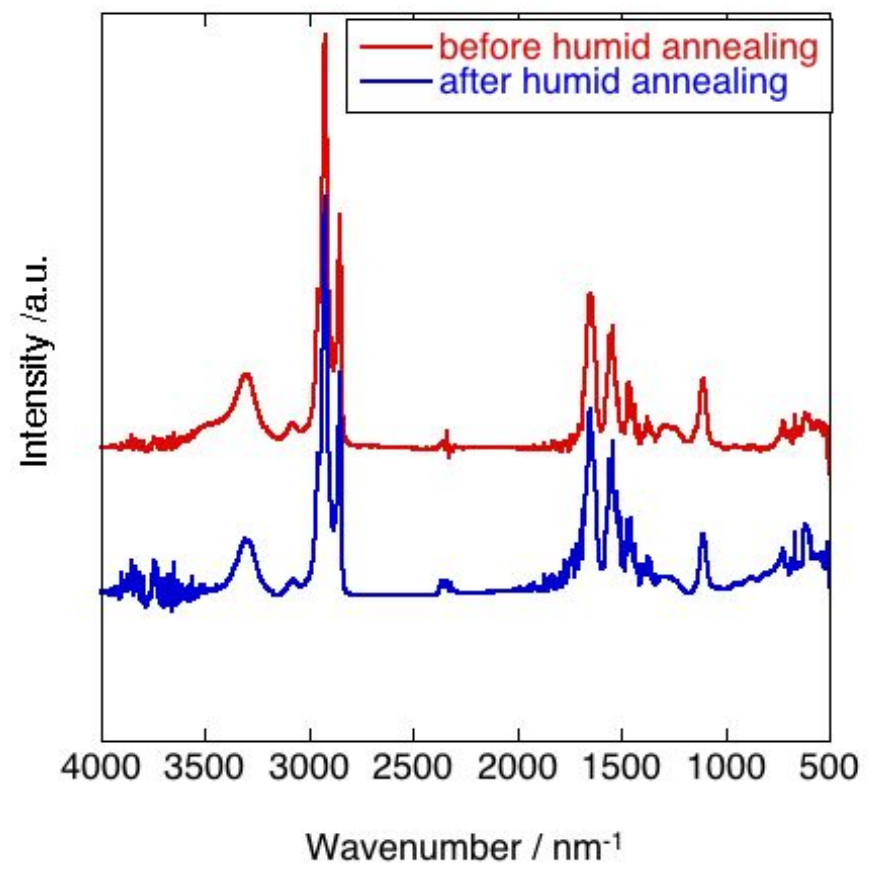

Figure S4 FT-IR spectra of $\mathrm{pDDA}_{80}-b$-PEG4 $4_{5}$ film before and after humid annealing 

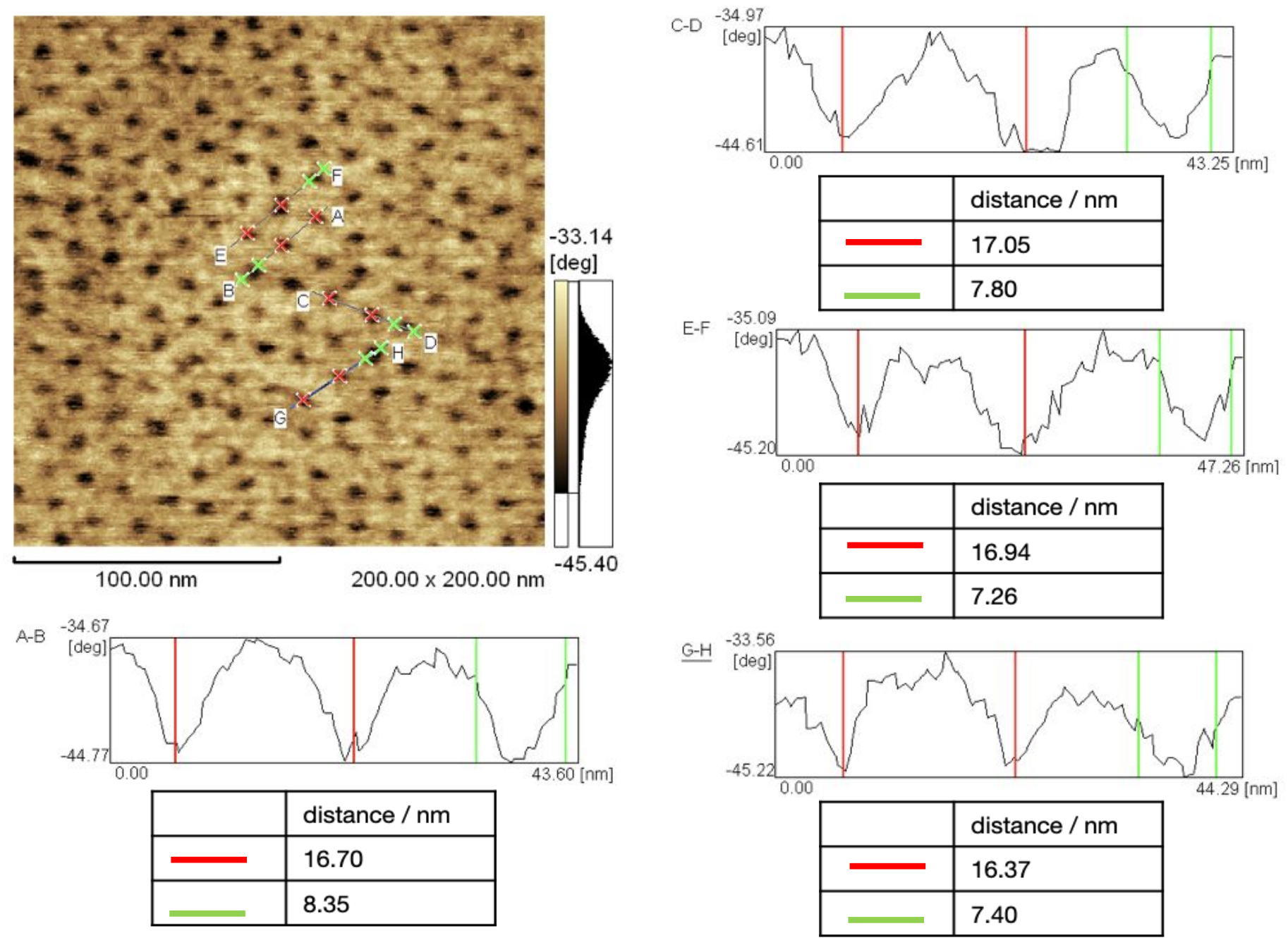

Figure S5 AFM analysis for $\mathrm{pDDA}_{80}-b-\mathrm{PEG}_{45}$ film 


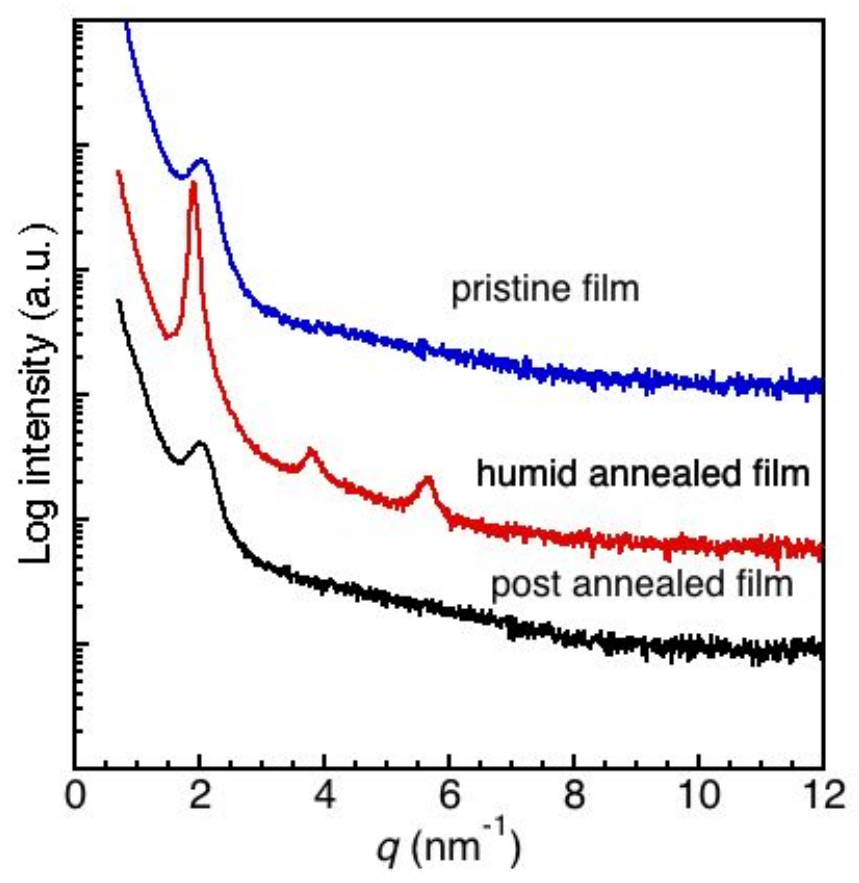

Figure S6 XRD patterns for $\mathrm{pDDA}_{80}-b-\mathrm{PEG}_{45}$ film for pristine (blue), humid annealed (red) and post annealed (black) film. Post annealed film was prepared by annealing the humid annealed film at $90{ }^{\circ} \mathrm{C}$ for $1 \mathrm{~h}$.

(a)

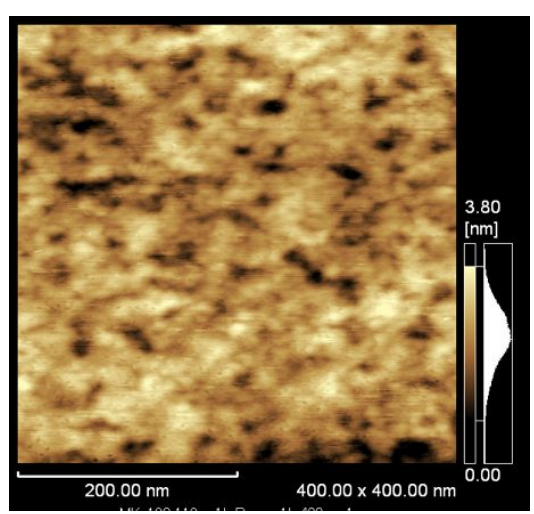

(b)

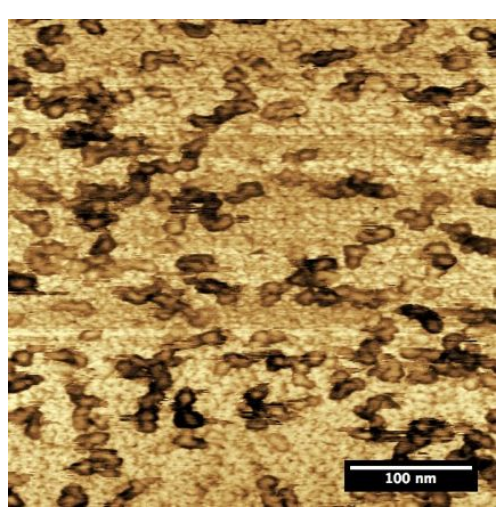

Figure S7 AFM height (a) and phase (b) image for post annealed $\mathrm{pDDA}_{80}-b-\mathrm{PEG}_{45}$ film 